\title{
Solving the maximum subsequence sum and related problems using BSP/CGM model and multi-GPU CUDA
}

Anderson C. Lima ${ }^{1+*}$ (D), Rodrigo G. Branco ${ }^{1 \dagger}$, Samuel Ferraz ${ }^{1}$, Edson N. Cáceres ${ }^{1}$, Roussian A. Gaioso ${ }^{2 \dagger}$, Wellington S. Martins ${ }^{2}$ and Siang W. Song ${ }^{3}$

\begin{abstract}
Background: The maximum subsequence problem finds a contiguous subsequence of the largest sum of a sequence of $n$ numbers. Solutions to this problem are used in various branches of science, especially in applications of computational biology. The best sequential solution to the problem has an $O(n)$ running time and uses dynamic programming. Although effective, this solution returns little information and disregards the existence of more than a maximum subsequence sum. Particularly in DNA analysis, if we find all maximum subsequence sums, we will also find all the possible pathogenicity islands, which are stretches with high possibility of causing some diseases.

Methods: We present new Bulk Synchronous Parallel/Coarse-Grained Multicomputer (BSP/CGM) parallel algorithms, which consider the existence of more than one subsequence of maximum sum, and are able to find solutions to three problems: the longest maximum subsequence sum, the shortest maximum subsequence sum, and the number of disjoint subsequences of maximum sum. To the best of our knowledge, there are no parallel BSP/CGM algorithms for the related problems. Taking advantage of the advent of general purpose graphics processing unit (GPGPU), we implemented our algorithms on multi-GPU with Compute Unified Device Architecture (CUDA) and, for comparison purposes, MPI and OpenMP implementations have also been developed.
\end{abstract}

Results: The algorithms presented good speedups, as confirmed by experimental results. They use $p$ processors and require $\mathrm{O}(n / p)$ parallel time with a constant number of communication rounds for the algorithm of the maximum subsequence sum and $\mathrm{O}(\log p)$ communication rounds, with $\mathrm{O}(\mathrm{n} / \mathrm{p})$ local computation per round, for the algorithms of the related problems.

Conclusions: We concluded that our algorithms for the maximum subsequence sum and related problems are unique and effective. We also believe that the BSP/CGM model can guide parallel implementations in modern architectures such as GPGPU/CUDA. As future work, we intend to extend these results to arrays with higher dimensions and compute all maximal subsequences in a given interval.

Keywords: Parallel algorithms, Multicore, GPGPU, BSP/CGM, Maximum subsequence sum problem

${ }^{*}$ Correspondence: anderson.correa.lima@gmail.com

A short version of this paper was presented at the ICCS-2015 [8]

${ }^{\dagger}$ Equal Contribution

${ }^{1}$ Faculdade de Computação da Universidade Federal de Mato Grosso do Sul,

Cidade Universitária, C.P: 549 Campo Grande, MS, Brasil

Full list of author information is available at the end of the article 


\section{Background}

Given a sequence of $n$ numbers, the task of finding the contiguous subsequence, with maximum sum over all subsequences of the given sequence, is called the maximum subsequence sum problem [2]. We also refer to this problem as the $1 \mathrm{D}$ maximum subsequence sum problem. The solution of this problem arises in many areas of science, such as computational biology, where many applications require the solution of the maximum subsequence sum problem. Among these, finding regions of DNA that are rich or poor in nucleotides $G$ and $C$ (CG-content). The GC-content is especially important in the search for pathogenicity islands [9]. Another biological application is the identification of transmembrane domains in protein sequences. This is an important application and represents one of the important tasks to understand the structure of a protein or the membrane topology [12].

The best sequential algorithm for the maximum subsequence sum problem, has $\mathrm{O}(n)$ time complexity [2]. Despite being a simple and fast algorithm, it yields little information about the maximum subsequence sum. The output returns only the value of the maximum subsequence sum. The algorithm does not consider the length (number of elements) and existence of more than one maximum subsequence sum. The pseudocode is illustrated in Algorithm 1.

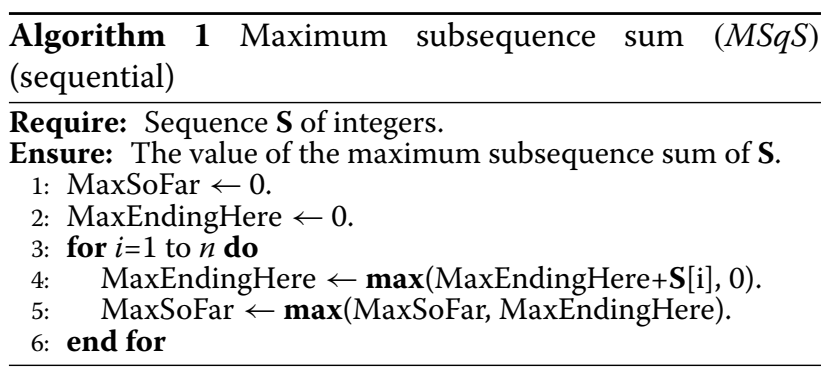

Previous works have reported good parallel solutions for the maximum subsequence sum problem. Qiu and Akl presented algorithms for the 1D (subsequence) and 2D (subarray) versions of the problem [11]. Their algorithms work on interconnection networks (hypercube and star) of length $p$, using $\mathrm{O}(n / p+\log p)$ parallel time with $p$ processors in version $1 \mathrm{D}$ [11]. Zhaofang Wen presented a parallel random access machine (PRAM) algorithm using $\mathrm{O}(\log n)$ parallel time with $\mathrm{O}(n / \log n)$ processors [16]. Perumalla and Deo also presented a PRAM algorithm with the same time complexity and number of processors [10]. A Bulk Synchronous Parallel/Coarse-Grained Multicomputer (BSP/CGM) algorithm for this problem was presented by Alves et al. using $\mathrm{O}(n / p)$ parallel time with $p$ processors and a constant number of communication rounds [1].
In this paper, we revisit the maximum subsequence sum problem and propose solutions to three related problems: the longest maximum subsequence sum, the shortest maximum subsequence sum, and the number of disjoint subsequences of maximum sum.

As far as we know, there are no parallel BSP/CGM algorithms for these three problems. The basis of our solution involves several variations of prefix sum in parallel [7].

The algorithms use $p$ processors and require $\mathrm{O}(n / p)$ parallel time with a constant number of communication rounds for the algorithm of the maximum subsequence sum and $\mathrm{O}(\log p)$ communication rounds, with $\mathrm{O}(n / p)$ local computation per round, for the algorithms of the related problems.

In order to show the efficiency not only in theory but also in practice, the algorithms were implemented on a machine with multiple GPUs using compute unified device architecture (CUDA). We also implemented the algorithms with MPI and OpenMP.

This paper is organized as follows. The "Methods" section defines the basic and related problems, discusses the extension of a solution already known, and presents our proposed BSP/CGM algorithms. The implementations and results are presented in the "Results" section.

Finally, the "Conclusions" section presents the conclusions and future work.

\section{Methods}

\section{The problems}

The basic problem of the 1D maximum subsequence sum may be defined formally as follows. For simplicity we consider the numbers to be integers. Consider a sequence $X_{n}=\left(x_{1}, x_{2}, \ldots, x_{n}\right)$ of $n$ integers. A subsequence is any contiguous segment $\left(x_{i}, \ldots, x_{j}\right)$ of $X_{n}$, where $1 \leq i \leq$ $j \leq n$. The $1 \mathrm{D}$ maximum subsequence sum problem is to determine, among all possible subsequences, the subsequence $M=\left(x_{i}, \ldots, x_{j}\right)$ that has the maximum sum $\left(\sum_{k=i}^{j} x_{k}\right)$ [2]. In the sequence represented in Fig. 1, there are three disjoint subsequences of maximum sum. The first consists of elements $\left(x_{1}, x_{2}\right)$ with size 2 ; the second is represented by the element $\left(x_{4}\right)$ with size 1 ; the third consists of the elements $\left(x_{6}, x_{7}, x_{8}, x_{9}\right)$ with size 4 . All have sum equal to 15 .

In a given sequence of integers, like sequence $X$ illustrated in Fig. 1, there might be more than one maximum subsequence sum. In this context, at least three new problems arise: the longest maximum subsequence sum, the shortest maximum subsequence sum, and the number of disjoint subsequences of maximum sum. In the first two problems, the size is related to the number of elements that make up the subsequences. 


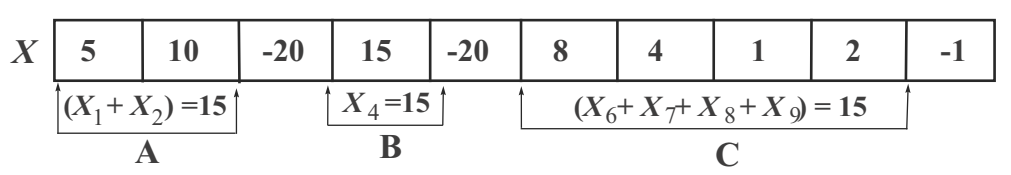

Fig. 1 The three subsequences of maximum sum of $X$ with the same sum [8]

\section{Computational model}

In this work we use the BSP/CGM parallel computation model $[3,15]$. It consists of a set of $p$ processors, each having a local memory of size $\mathrm{O}(n / p)$, where $n$ is the problem size.

The BSP/CGM parallel computing model is a realistic model where special attention is given to minimize communications overheads. This model is particularly suitable nowadays in parallel machines where the overall computation speed is considerably larger than the overall communication speed.

An algorithm in this model performs supersteps (a series of rounds), alternating well-defined local computation and global communication phases, separated by a synchronization barrier. The cost of communication considers the number of rounds required. The implementation of a BSP/CGM algorithm generally presents good results with performance similar to that predicted in its theoretical analysis [4]. Since the MPI library is designed for distributed memory environments, a BSP/CGM algorithm can be mapped into an MPI implementation using the message resources of this library. On the other hand, for the implementation of a BSP/CGM algorithm on a general purpose graphics processing unit (GPGPU) with CUDA, we need to establish the correspondence of computations and communications concepts in this environment. In this context, the supersteps of the BSP/CGM model are represented by sequential invocations of each CUDA kernel. Furthermore, we associate the set of processors of the BSP/CGM model with the set of CUDA streaming multiprocessors (SMs). Figure 2 illustrates our suggestion for this process.

\section{The extended solution}

In 2004, Alves et al. [1] presented a BSP/CGM algorithm for the maximum subsequence sum problem. This algorithm is efficient, but like the previous solutions, it does not consider the existence of more than a subsequence of maximum sum. Furthermore, since the algorithm works in a distributed environment, it performs a series of compressions that change the input sequence. This change causes difficulty in the search of more than one subsequence. On the other hand, using the ideas of the PRAM algorithm proposed by Perumalla and Deo [10], in this work we devise a new BSP/CGM parallel algorithm for this problem that uses the final output to compute the shortest/longest maximum subsequences and the total number of disjoint maximum subsequences. Figure 3 illustrates an example of the output array of Perumalla and Deo's algorithm [10]. Through the output array, we can find all disjoint subsequences of the maximum subsequence sum problem. In this example, there are three disjoint maximum subsequences with different sizes. Next we present new BSP/CGM algorithms to solve the $1 \mathrm{D}$ maximum subsequence sum problem, the shortest/longest maximum subsequences, and the total number of maximum subsequences.

\section{The BSP/CGM algorithm for the maximum subsequence sum}

We designed a BSP/CGM solution (see Algorithm 2) that solves the basic problem of maximum subsequence sum. In the algorithm, the arrays PSUM and SSUM mean prefix sum and suffix sum, respectively. We compute the suffix maxima of PSUM and prefix maxima of SSUM and store the results in the arrays SMAX and PMAX, respectively. The output of Algorithm 2 is an array $\mathbf{M}$ shown in Fig. 3.

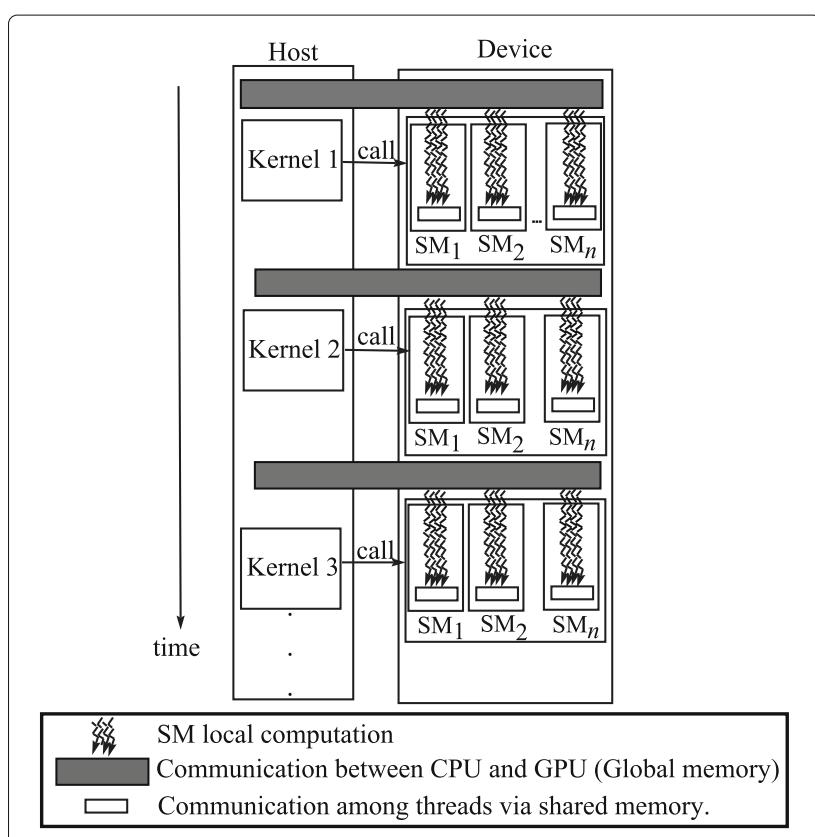

Fig. 2 Correspondence between the BSP/CGM and the GPGPU model 


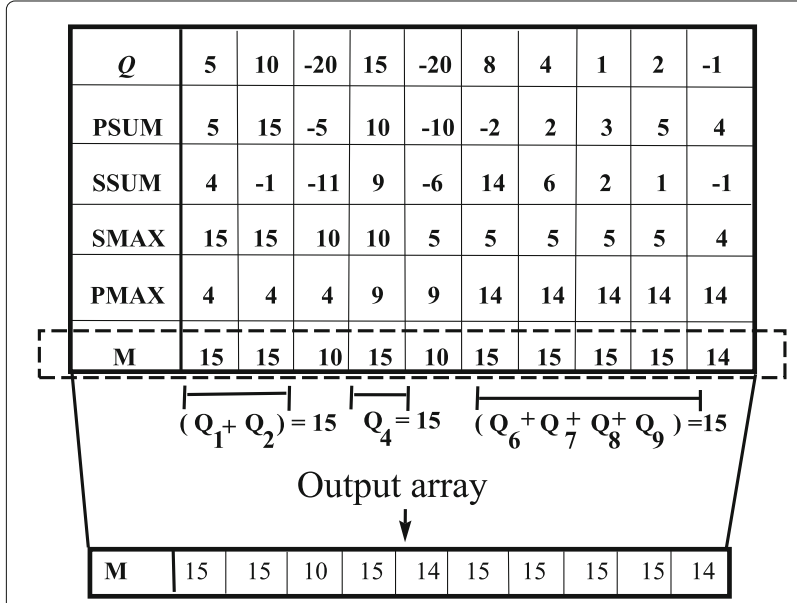

Fig. 315 is the highest value and represents the maximum sum of $\mathbf{Q}$ [8]

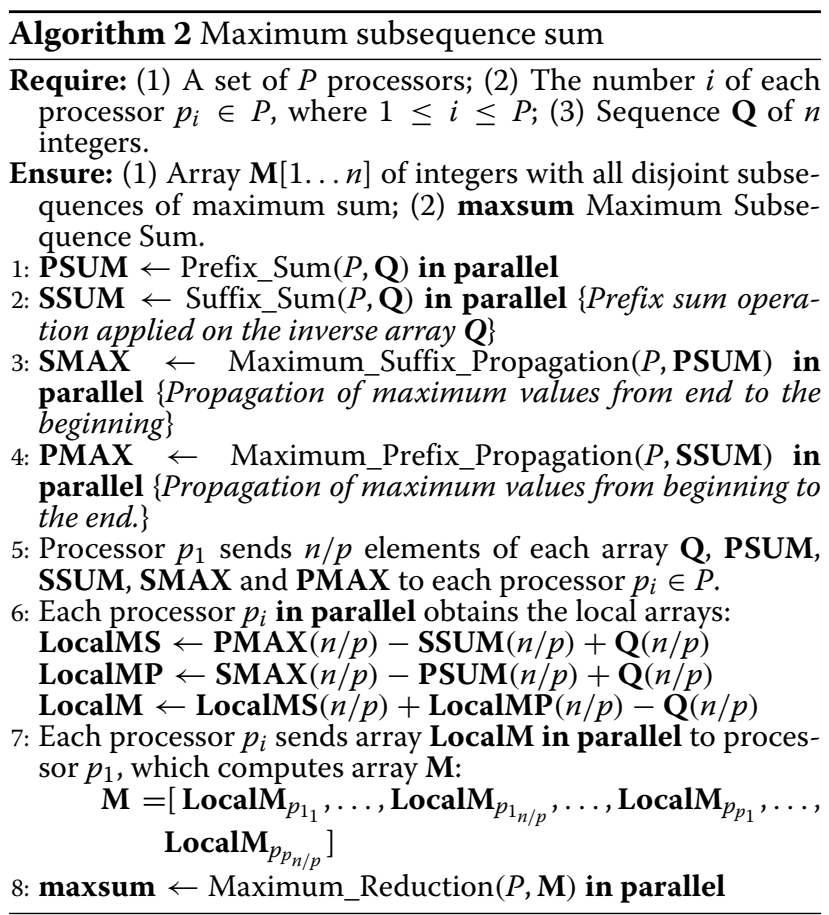

Two operations are performed in the algorithms "Maximum_Suffix_Propagation and Maximum_Prefix_ Propagation" (they are called from Algorithm 2), both consisting of a maximum propagation. The first operation occurs in the local array of each processor. In this case, higher values are propagated, replacing the smaller values. The replacement is performed until a (new) higher value is found, which then starts a new maximum propagation, as illustrated in Fig. 4. The second operation occurs between each processor and the greater value of the maximum values of the subsequent/precedent processors, as illustrated in Fig. 5. For the sake of simplicity, we present only the "Maximum_Suffix_Propagation" (Algorithm 3). The main difference of the algorithms is the direction of propagation. For the complete explanation about the variables (PSUM, SSUM, PMAX, SMAX, LocalMP, LocalMS, and LocalM), please see [10].

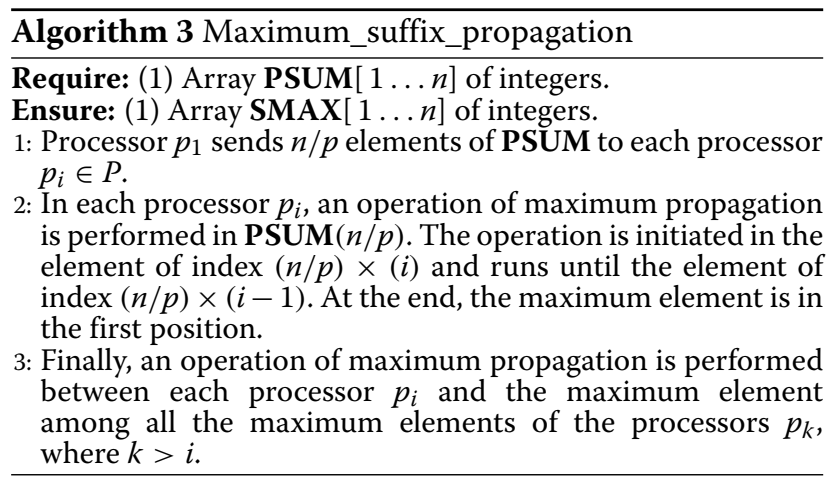

\section{Correctness and complexity}

Besides the fact that we use only $p$ processors, the steps of our main BSP/CGM algorithm can be easily derived from the PRAM algorithm [10]. Using BSP/CGM parallel prefix algorithms, steps 1 and 2 can be computed using $p$ processors in $\mathrm{O}(n / p)$ time and $\mathrm{O}(\log p)$ communication rounds. For steps 3 and 4 , we use Algorithm 3, requiring $p$ processors with $\mathrm{O}(n / p)$ time and $\mathrm{O}(\log p)$ communication rounds. Steps 5 to 7 can be computed using $p$ processors with $\mathrm{O}(n / p)$ time and a constant number of communication rounds. Finally, using BSP/CGM parallel maximum reduction algorithm, step 8 is computed using $p$ processors with $\mathrm{O}(n / p)$ time and $\mathrm{O}(\log p)$ communication rounds. Therefore, Algorithm 2 computes the maximum subsequence sum correctly using $p$ processors in $\mathrm{O}(n / p)$ time with $\mathrm{O}(\log p)$ communication rounds.

\section{The BSP/CGM algorithms for the related problems}

We also developed BSP/CGM algorithms to solve three problems related to the maximum subsequence sum, that is, (1) the longest maximum subsequence sum, (2) the shortest maximum subsequence sum, and (3) the number of disjoint subsequences of maximum sum. The output of Algorithm 2 is the starting point of these three algorithms. For simplicity, the strategies of these algorithms are listed in only one algorithm, represented here by Algorithm 4.

First, using the array TransArray, we mark all the elements of array $M$ that are equal to maxsum.

Then, we compute the array SegScan that stores the regions where the elements of $M$ have the same value. We call this operation Segmented_Scan. We define this operation as follows: if $\mathbf{M}[i-1]=\mathbf{M}[i]$ then $\mathbf{S e g S c a n}[i] \leftarrow$ $\operatorname{SegScan}[i-1]+1$; otherwise, $\operatorname{SegScan}[i] \leftarrow 1$. We define $\operatorname{SegScan}[0] \leftarrow 1$. 


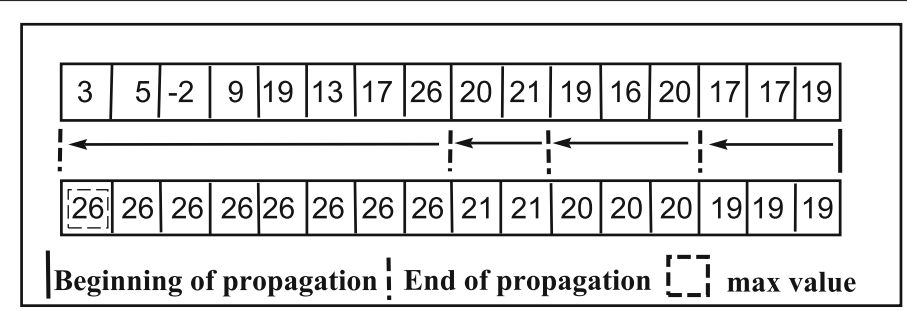

A - Example of maximum suffix propagation in each processor (Algorithm 3)

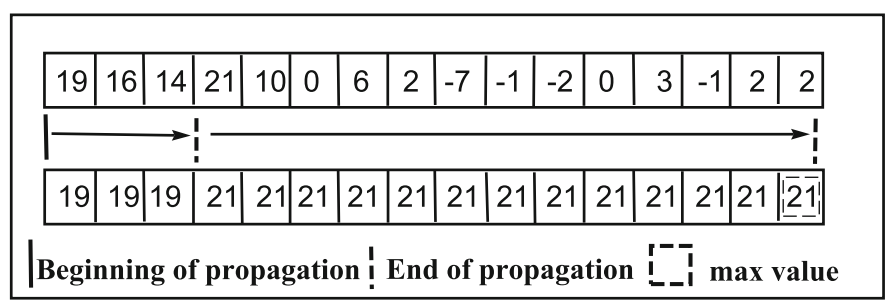

B - Example of maximum prefix propagation in each processor (Algorithm 4)

Fig. 4 Local operations

Since we are searching the regions in $M$ where we have maximum values, we perform a bitwise operation with the SegScan and TransArray arrays. This operation changes the SegScan array and all the elements in this array that do not correspond to the elements in $M$ that represent the maximum subsequences become equals 0 . The subarrays in SegScan that are not null are enumerated and represent the maximum sum subsequences.

The maximum subsequences and their respective sizes are easily found using parallel BSP/CGM prefix/suffix sum, reduction, and other basic algorithms.

\section{Correctness and complexity}

In order to find the maximum subsequences in $M$, we use basic BSP/CGM algorithms for prefix/suffix sum, Segmented Scan and Bitwise And Operation. Efficient BSP/CGM parallel algorithms for these problems can be found in Dehne at al. [4]. Figure 6 illustrates the relationship among the internal routines listed by Algorithm 4 and the solutions that can be drawn from them. The starting point is the output of Algorithm 2. Algorithm 4 computes correctly and returns the three expected values.

Using BSP/CGM reduction algorithms, the internal routines of Algorithm 4 associated with the sizes of maximum subsequence sum can be computed using $p$ processors in $\mathrm{O}(n / p)$ time and $\mathrm{O}(\log p)$ communication rounds. Hence, the combination of Algorithms 2 and 4 yields the solution in $\mathrm{O}(n / p)$ time with $\mathrm{O}(\log p)$ communication rounds.

\section{Results}

The main advantage of using the BSP/CGM model to design parallel algorithms is that when implemented in real environments, they have an expected behavior as stated by their theoretical analysis. In this work, we implemented our algorithm using both distributed and shared memory environments. The results showed that the execution times were compatible with those foreseen by the theoretical analysis.

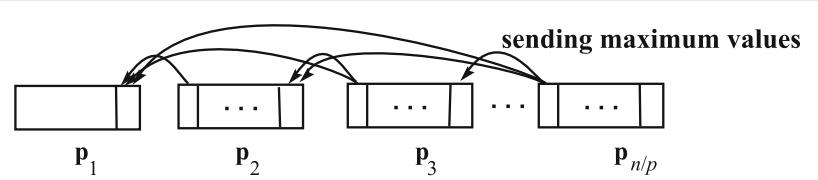

A = Maximum suffix propagation between processors (Algorithm 3)

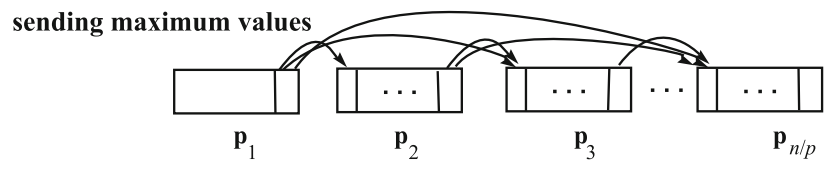

B = Maximum prefix propagation between processors (Algorithm 4)

Fig. 5 Global operations 


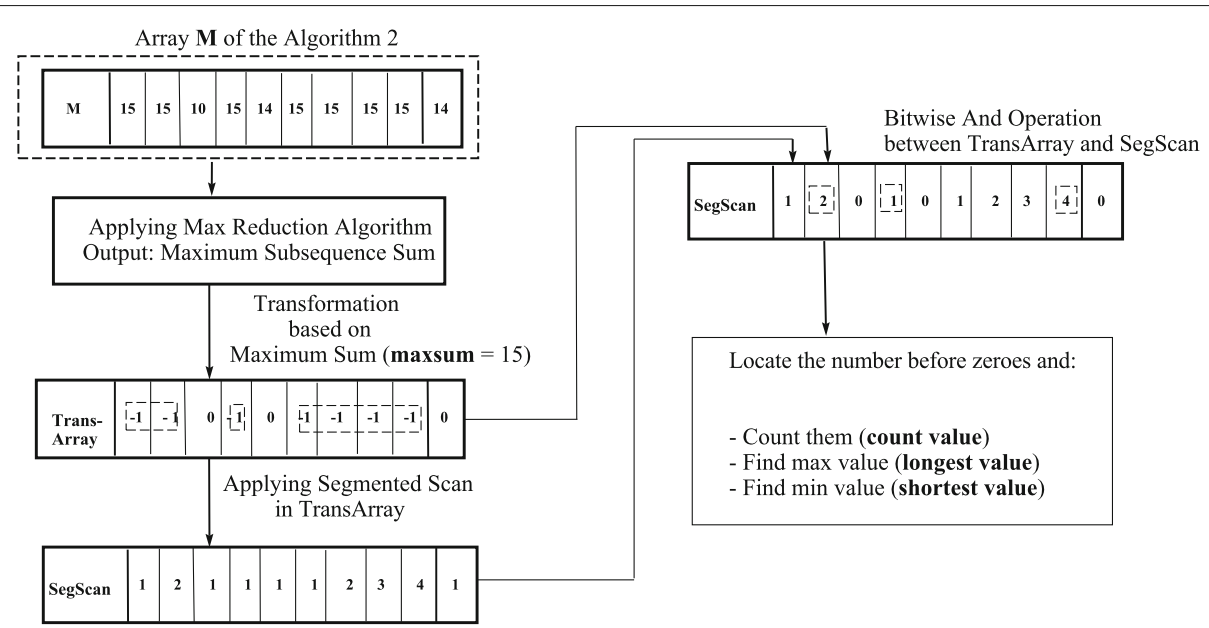

Fig. 6 Problems that can be solved from the output array $M$

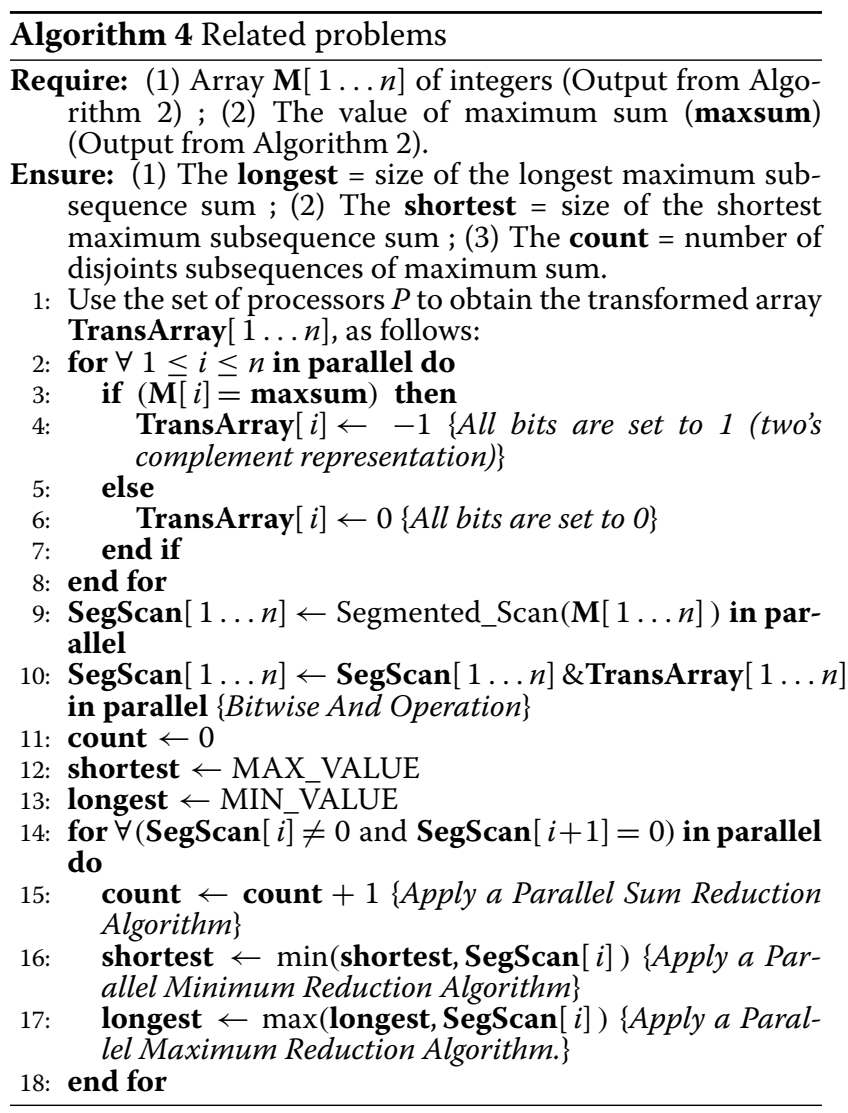

We used a 32-node cluster of workstations and the message passing interface (MPI) to implement our algorithms in a distributed memory environment. The shared memory environment was tested with several configurations. The open multi-processing (OpenMP) implementation used workstations with 2/4/6 cores. The GPGPU/CUDA version of the algorithm was implemented using one GPU and four GPUs. Previous works have shown the good performance of BSP/CGM algorithms when implemented on clusters of workstations [1]. In this work, we use four different computing systems with shared memory, which allow us to confront our shared memory implementations against a similar distributed memory implementation. For comparison purpose, the speedups were computed using as reference the time spent by the sequential implementation in a host of the computing system were the respective parallel algorithms where executed. All the implementations showed competitive speedups.

\section{Computational resources}

We have run the experiments on five different computing systems (Table 1), with shared and distributed memory. Four of those computing systems (CS-1, CS-2, CS-3, and CS-5) use shared memory and have at least one CUDA GPU (one of them has four identical GPUs) with different specifications, which allow us to check the consistency

Table 1 Platforms-general specs

\begin{tabular}{llllll}
\hline ID & Processor & Clock & Cores/threads & RAM/cache & Linux/dist./GCC \\
\hline CS-1 & Xeon E5-2620 & 2.00 & $6 / 12$ & $16 / 15$ & CentOS 7.2/3.10.0/4.8.5 \\
CS-2 & Core i7-3770 & 3.40 & $4 / 8$ & $8 / 8$ & Ubuntu 12.04/3.2.0-37/4.6.3 \\
CS-3 & Core2 Quad Q9550 & 2.83 & $4 / 4$ & $4 / 6$ & Ubuntu 15.04/3.19.0-15/4.9.2 \\
CS-4 & Xeon E5620 & 2.40 & $4 / 8$ & $8 / 12$ & CentOS 6.8/2.6.32/4.4.7 \\
CS-5 & Core i7-4500U & 1.80 & $2 / 4$ & $8 / 4$ & Ubuntu 15.04/3.19.0/4.9.2 \\
\hline
\end{tabular}


Table 2 Platforms-CUDA specs

\begin{tabular}{|c|c|c|c|c|c|c|c|}
\hline ID & GPU (NVidia) & \# GPUs & RAM & \# SMs & Cuda cores & Clock & GPU arch/SDK \\
\hline $\mathrm{CS}-1$ & GTX TITAN Black & 4 & 6 & 15 & 2880 & 889 & $3.5 / 7.5$ \\
\hline $\mathrm{CS}-2$ & GTX 680 & 1 & 2 & 8 & 1536 & 1006 & $3.0 / 4.2$ \\
\hline $\mathrm{CS}-3$ & GTX 460 v2 & 1 & 1 & 7 & 336 & 778 & $2.1 / 7.5$ \\
\hline CS-5 & GT 720M & 1 & 2 & 2 & 192 & 797 & $2.1 / 7.5$ \\
\hline
\end{tabular}

and performance of our algorithms using shared memory environment. One of the computing systems (CS-4) is a cluster of 32 nodes connected with a Myrinet switch using $10 \mathrm{~Gb} / \mathrm{s}$ Ethernet.

The technical specifications of the computing systems are depicted in Tables 1, 2, and 3. All the processors use 64-bit architecture. The clock frequency is given in $\mathrm{GHz}$, the cache memory in $\mathrm{MB}$, and the RAM memory in GB.

\section{Execution methodology}

We used randomly generated data for input arrays varying from $2^{20}$ to $2^{29}$ elements. For each input, we ran the experiment 20 times and measured the average running time. To make sure that such an average is representative of the expected value, we apply the Shapiro-Wilk [14] statistical test to the 20 times obtained, and we tolerate the discarding of five values, thereby resulting in a minimum of 15 and a maximum of 20 valid values to compute the average. The result of the statistical test ( $P$ value) has to be greater than $\alpha>0.05$, so that we can assert with significance level of $5 \%$ that the sample derives from a normal population. In case the statistical test gives $\alpha \leq 0.05$, the sample is discarded and new running times are collected for that input.

The algorithms were tested in the distributed memory environment CS-4 using MPI. In this environment, we can specify the number of nodes and their respective processors to be allocated for the execution, in the form of $N X$ : $P Y$ ( $X$ nodes, with $Y$ processors each).The configuration N32 : $P 8$ means that we used 32 nodes of the cluster, each with 8 threads, with a total of 256 MPI processes. We executed our algorithm with the following configurations: $N 16: P 1, N 16: P 2, N 16: P 4, N 16: P 8, N 32: P 1$, $N 32: P 2, N 32: P 4$, and $N 32: P 8$.

In the shared memory environment, we used OpenMP and CUDA. The CUDA implementation of the algorithms were executed on CS-1, CS-2, CS-3, and CS-5 platforms. In the CS-1 platform, we also varied the number of GPUs, where we used 1,2, and 4 GPU configurations. The OpenMP implementation was executed in all platforms.

Table 3 Platforms-MPI specs

\begin{tabular}{llllll}
\hline ID & Cluster & \# Nodes/threads & \# MPI proc. & mpicc & Comm. \\
\hline CS-4 & $\begin{array}{l}\text { Cluster Rocks } \\
\text { 6.0 (Mamba) }\end{array}$ & $46 / 8$ & 368 & 4.4 .7 & Myrinet \\
& & & & $10 \mathrm{~Gb} / \mathrm{s}$ \\
\hline
\end{tabular}

We tested the OpenMP implementation with different numbers of threads. In all platforms, we used 1, 2, and 4 threads, while we also tested with 8 threads in the CS-1, CS-2, and CS-4 and with 12 threads in the CS-1.

A sequential version of the algorithm, with the related problems, was implemented using C. This implementation was executed in all platforms. The speedups of the parallel versions were computed using the execution time of the sequential implementation of the algorithms in the respective platform as reference.

Due to the exponential variation of the input values (with $2^{20} \leq n \leq 2^{29}$ ), all the curves use the logarithmic scale (base 2) as the horizontal axis. Vertical axis (runtime and speedup) uses logarithmic scale (base 10) for better presentation and visualization.

\section{Results}

Now we present the results that were obtained using the five computing systems. First we present the execution times of the sequential version of the algorithms. As we stated above, we implemented it on all platforms in order to compute the respective speedup in each computer system.

\section{Sequential implementation}

We developed a sequential version of the Perumalla and Deo parallel algorithm [10], and we extended the sequential version for solving the related problems. The complete algorithm has $\mathrm{O}(n)$ time complexity. It was implemented on all platforms.

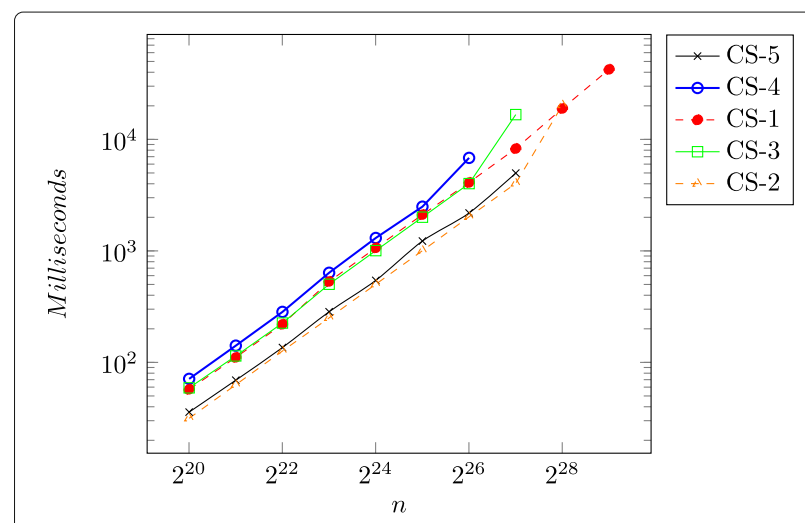

Fig. 7 Running times (milliseconds) of sequential implementation 
Table 4 Running times (milliseconds) of sequential implementation

\begin{tabular}{llllll}
\hline$n$ & CS-5 & CS-4 & CS-1 & CS-3 & CS-2 \\
\hline $2^{20}$ & 35.811 & 71.143 & 57.314 & 59.100 & 31.517 \\
$2^{21}$ & 69.225 & 141.295 & 110.836 & 114.250 & 62.986 \\
$2^{22}$ & 135.859 & 283.747 & 219.730 & 225.275 & 126.487 \\
$2^{23}$ & 284.376 & 636.522 & 531.464 & 502.136 & 250.795 \\
$2^{24}$ & 543.696 & 1305.605 & 1063.938 & 1005.549 & 501.978 \\
$2^{25}$ & 1225.326 & 2492.813 & 2114.072 & 2005.769 & 1004.222 \\
$2^{26}$ & 2186.893 & 6825.944 & 4105.771 & 4012.655 & 2022.964 \\
$2^{27}$ & 4985.319 & - & 8272.913 & $16,682.755$ & 4030.542 \\
$2^{28}$ & - & - & $18,931.466$ & - & - \\
$2^{29}$ & - & - & $42,466.062$ & & $20,327.877$ \\
\hline
\end{tabular}

Figure 7 and Table 4 show the behavior of the algorithm on each platform. We observe that when the input size reaches the resource limits of the platform, the execution results stop obeying the expected curve of the execution times. This phenomenon is called Thrashing [5]. It occurs because of the constant paging, which degrades the complete system performance. In some cases, it freezes the system. In some tests, the operating system killed the process and we could not collect the result. In these cases, if the time was collected, it would be equivalent or worse than that when the thrashing phenomenon occurs.

\section{MPI implementation}

We developed a version of the BSP/CGM algorithms for distributed memory. This version was implemented with MPI and uses the resources of MPI which includes a number of library functions for communication and synchronization among all members of a process group.

The sequence is divided in $p$ subsequences $s_{i}, 1 \leq i \leq p$ of size $n / p$. Each processor $p_{i}(1 \leq i \leq p)$ receives the subsequence $s_{i}$ and compute the algorithm locally. The partial solutions (boundaries) are exchanged using built-in functions of the MPI. The details of the code can be seen in https://github.com/rodrigogbranco/extendedmss.

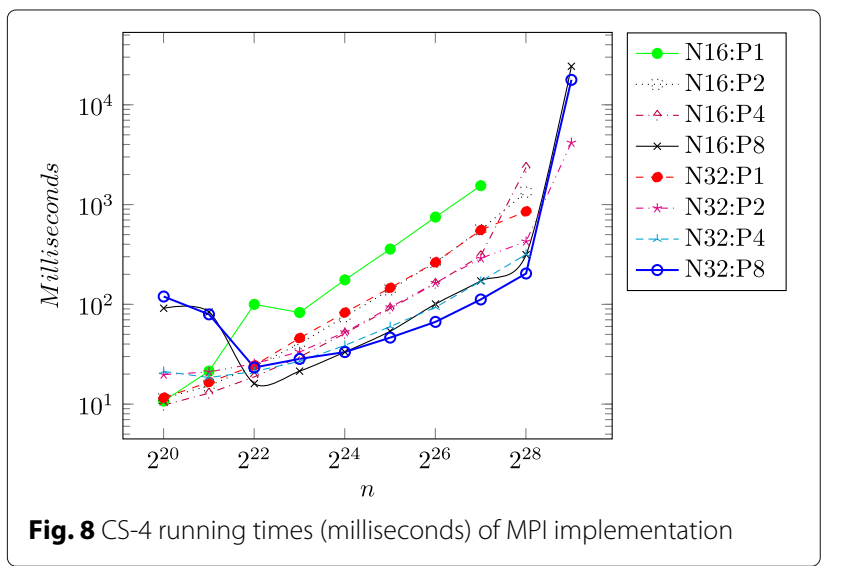

Figure 8 and Table 5 show the average execution times. We executed the MPI implementation using 16 and 32 nodes with different numbers of threads.

We can see that when the input is not big enough, there is a communication overhead. This depends on the number of used nodes and impacts the execution times. After a given size of the input, the execution times present a linear behavior (in each processor the time complexity is $\mathrm{O}(n / p))$. When the size of the input is too big, the thrashing phenomenon occurs again. In some cases, such as $N 16: P 1$, the operating system/cluster management aborted the application with input sizes of $n=2^{28}$ and $n=2^{29}$.

\section{OpenMP implementations}

Using the ideas of the distributed memory BSP/CGM algorithms that were implemented with MPI, we implemented them in a shared memory environment using OpenMP (Table 6 and Fig. 9). The main difference was that the message exchange among the processes (threads) was done in the memory of the workstations. We used the OpenMP directives to create and eliminate threads and shared arrays and schedule functions. When supported by the compilers, we used directives for array reduction. The details of the code can be seen in https://github.com/ rodrigogbranco/extendedmss.

The shared memory implementation of the BSP/CGM algorithms with OpenMP shows a linear curve on all platforms. This confirms the theoretical complexity of the BSP/CGM algorithms, which have $\mathrm{O}(n / p)$ time complexity in each computation round. The phenomenon of thrashing and the finalization of the execution for the operating system was also observed for some large inputs. Besides the good speedups that we obtained for the BSP/CGM algorithms with OpenMP, the execution times of the cluster platform (CS-4) were smaller. This leads us to explore another shared memory environment, the GPGPU with CUDA. 
Table 5 CS-4 running times (milliseconds) of MPI implementation

\begin{tabular}{lllllllll}
\hline$n$ & $N 16: P 1$ & $N 16: P 2$ & $N 16: P 4$ & $N 16: P 8$ & $N 32: P 1$ & $N 32: P 2$ & N32:P4 & N32:P8 \\
\hline $2^{20}$ & 10.730 & 11.403 & 9.788 & 91.368 & 11.597 & 19.765 & 21.263 & 119.741 \\
$2^{21}$ & 21.403 & 15.286 & 12.880 & 83.876 & 16.562 & 21.004 & 18.488 & 79.335 \\
$2^{22}$ & 99.957 & 23.938 & 18.761 & 16.064 & 24.395 & 25.461 & 21.225 & 23.279 \\
$2^{23}$ & 82.786 & 40.277 & 29.675 & 21.406 & 45.941 & 33.450 & 26.846 & 28.399 \\
$2^{24}$ & 175.851 & 75.340 & 51.073 & 33.305 & 82.706 & 52.493 & 38.760 & 33.242 \\
$2^{25}$ & 357.882 & 139.970 & 90.844 & 53.800 & 146.140 & 93.536 & 59.839 & 46.379 \\
$2^{26}$ & 748.831 & 263.430 & 161.765 & 100.714 & 263.629 & 164.268 & 93.531 & 66.719 \\
$2^{27}$ & 1546.020 & 559.685 & 307.145 & 171.782 & 555.407 & 288.917 & 170.073 & 111.906 \\
$2^{28}$ & - & 1331.303 & 2356.562 & 314.681 & 854.059 & 426.578 & 317.116 & 203.811 \\
$2^{29}$ & - & - & - & $24,301.072$ & - & 4174.692 & - & $17,766.883$ \\
\hline
\end{tabular}

\section{Multi-GPU implementation}

One of the goals of this work is to show that the BSP/CGM model is suitable for designing parallel algorithms for distributed and shared memory alike. Besides that, we also want to show that the BSP/CGM algorithms can be implemented in GPGPUs with good speedups. In order to verify the efficiency of the algorithm in the GPGPUs environment, we tested it with four different configurations. One of the configurations is a multi-GPGPU with four devices (GPUs).

Differently from the MPI and OpenMP environments, the implementation of a BSP/CGM algorithm using CUDA/GPGPU has a higher level of complexity. For this reason, in this section, we choose to present a step by step description of the parallel implementation of the 1D maximum subsequence sum and the related problems using CUDA/GPGPU. The details of the code can be seen in https://github.com/rodrigogbranco/extendedmss.

Figure 2 shows how we can map a BSP/CGM algorithm onto a GPGPU. The computation rounds are done by the SMs. The computation is executed by sets of blocks of threads. Each thread runs a copy of the same program in the GPGPU (kernel function).
We implemented our algorithm in the GPGPU using CUDA. With CUDA, a kernel function specifies the code to be executed by all threads in a parallel step (computation round). When a kernel is called, or launched, it is executed as a grid of thread blocks in parallel. Each thread block is scheduled separately for a SM. Threads within the same block are organized in units of 32 threads, called warps [13]. To maximize even further the performance, CUDA uses different memory types. Each memory is used for different purposes. The shared memory, for example, is used to exchange data between threads within a CUDA block [13]. When a kernel finishes its computation, we have a synchronization. After that, we have a new round or the program finishes.

First we describe the steps of our implementation that uses only one GPGPU.

As shown in Table 7, we used the Thrust Library [6] extensively, including the device vector (using thrust::device_vector) and host vector (using thrust::host vector) when implementing Algorithms 2 and 4 with CUDA. These types of vectors were chosen to simplify the use of optimized Thrust functions, and also the memory transfers between device-host and vice-versa.

Table 6 Running times (milliseconds) of OpenMP implementation

\begin{tabular}{llllll}
\hline$n$ & CS-5 4T & CS-4 8T & CS-1 12T & CS-3 4T & CS-2 8T \\
\hline $2^{20}$ & 23.917 & 28.162 & 13.353 & 27.371 & 9.412 \\
$2^{21}$ & 45.392 & 50.601 & 26.081 & 53.049 & 17.390 \\
$2^{22}$ & 91.215 & 100.928 & 46.429 & 107.229 & 34.647 \\
$2^{23}$ & 175.983 & 210.809 & 84.330 & 215.435 & 69.902 \\
$2^{24}$ & 332.776 & 471.320 & 157.892 & 457.911 & 137.247 \\
$2^{25}$ & 684.553 & 860.291 & 297.038 & 911.215 & 274.483 \\
$2^{26}$ & 1406.760 & 1620.361 & 614.651 & 1816.237 & 539.597 \\
$2^{27}$ & 3018.799 & - & 1355.126 & 5835.837 & 1103.898 \\
$2^{28}$ & 5855.649 & - & 2362.273 & - & 2188.595 \\
$2^{29}$ & - & - & 7082.654 & & $5,169,643.376$ \\
\hline
\end{tabular}




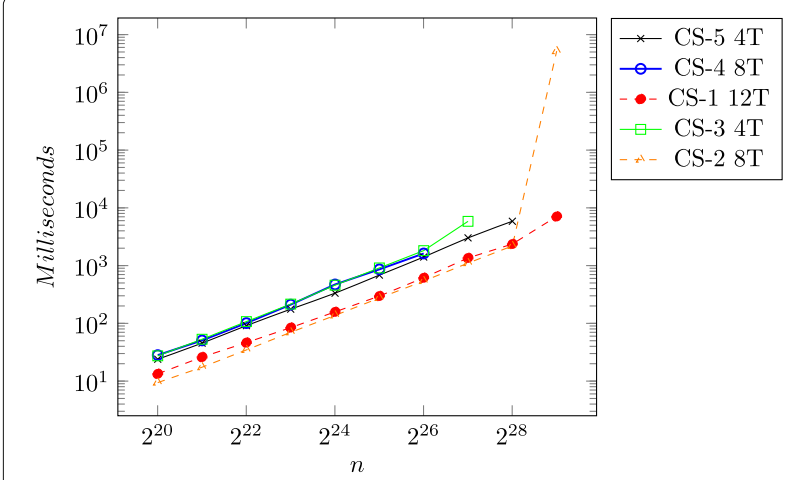

Fig. 9 Running times (milliseconds) of OpenMP implementation

Now we detail the CUDA implementation steps of Algorithms 2 and 4.

Steps 1 and 2 (Sum of Prefixes and Sum of Suffixes): In order to solve steps 1 and 2, we used thrust::inclusive_scan. We used the reverse iterator of thrust::device_vector to iterate and compute sum of suffixes transparently.

Steps 3 and 4 (Maximum Suffix Propagation and Maximum Prefix Propagation): These steps follow the same idea of steps 1 and 2 but using thrust::maximum rather than the default thrust::plus used on thrust::inclusive_scan.

Steps 5-9: To build the output array $M$, some arithmetic operations involving the initial four arrays were needed. For that, we used the function thrust::transform to operate individually on each element of array.

Table 7 Thrust and CUDA functions used by Algorithms 2 and 4

\begin{tabular}{|c|c|c|}
\hline Algorithm & Steps & Thrust function \\
\hline Algorithm 2 & (1) PSUM (2) SSUM & thrust::inclusive_scan \\
\hline Algorithm 2 & (1) PSUM (2) SSUM & $\begin{array}{l}\text { thrust::for_each (to correct } \\
\text { borders - multi-GPUs) }\end{array}$ \\
\hline Algorithm 2 & (3) SMAX (4) PMAX & thrust::inclusive_scan \\
\hline Algorithm 2 & (3) SMAX (4) PMAX & $\begin{array}{l}\text { thrust::for_each (to correct } \\
\text { borders - multi-GPUs) }\end{array}$ \\
\hline Algorithm 2 & (7) Compute array M & thrust::transform \\
\hline Algorithm 2 & (8) Maximum reduction & thrust::reduce \\
\hline Algorithm 4 & (2) Transformation & thrust::for_each \\
\hline Algorithm 4 & (7) Segmented scan & thrust::inclusive_scan_by_key \\
\hline Algorithm 4 & (8) Bitwise and operation & thrust::transform, thrust::find \\
\hline Algorithm 4 & (8) Bitwise and operation & $\begin{array}{l}\text { thrust::for_each (to correct } \\
\text { borders - multi-GPUs) }\end{array}$ \\
\hline Algorithm 4 & (12) Find related solutions & $\begin{array}{l}\text { findRelatedSolutions } \\
\text { (Custom Kernel) }\end{array}$ \\
\hline- & Synchronization & __syncthreads (threads) \\
\hline- & Synchronization & $\begin{array}{l}\text { cudaDeviceSynchronize } \\
\text { (blocks and device-host) }\end{array}$ \\
\hline
\end{tabular}

Step 10: To solve this step, we used thrust::reduce using thrust::maximum.

At the beginning of Algorithm 4, we built the TransArray using thrust::for_each, with a custom function operation to manipulate the individual elements. So, in that function we passed the maxsum and, if the element was equal to it, then it would receive -1 value and 0 otherwise. We built a new array using TransArray, applying the Segmented_Scan offered by Thrust through thrust::inclusive_scan_by_key (see Fig. 6).

With both TransArray and SegScan ready, we applied the bitwise and operation (\&) using thrust::transform and thrust::bit_and. Finally, we built a CUDA kernel to process the last part of Algorithm 4 (quantity of maximum subsequence sum, longest subsequence, and shortest subsequence).

Our CUDA implementation with more than one GPU works on the same host. Therefore, in order to manage each GPU transparently, we used OpenMP, and each thread was responsible for managing its own GPU. It is important to note that here OpenMP is being used only to manage the GPUs and run the synchronization among them through the function cudaDeviceSynchronize() (GPU-host synchronization), followed by the directive \#pragma omp barrier (synchronization among threads).

When the algorithm was implemented in a multi-GPU system, we had to take care of some details. After the end of some steps of a GPU we need to do some extra work, since each GPU works with a $n /(\# g p u s)$ partition of the array $\mathbf{M}[1 \ldots n]$. The same occurs for the arrays PSUM and SSUM. After the GPU finalizes each of steps 1 and 2 of Algorithm 2, they need to correct their array borders. Those corrections can be done by using the shared memory of the host with the function thrust::for_each. At the end of steps 3 and 4, the first element of arrays $P M A X$ and SMAX may need to be corrected too. This also is done with the function thrust::for_each. Algorithm 4 uses the function thrust::for_each after step 8 to correct the

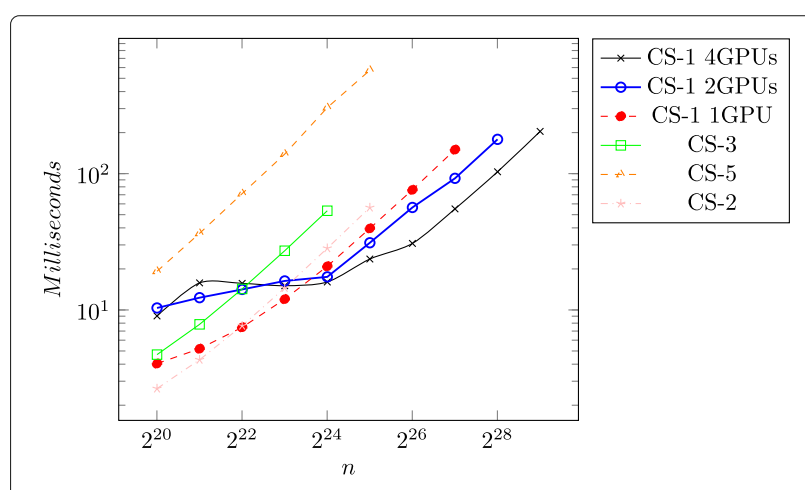

Fig. 10 Running times (milliseconds) of CUDA implementation 
Table 8 Running times (milliseconds) of CUDA implementation

\begin{tabular}{|c|c|c|c|c|c|c|}
\hline$n$ & CS-1 4GPUs & CS-1 2GPUs & CS-1 1GPU & $\mathrm{CS}-3$ & CS-5 & CS-2 \\
\hline $2^{20}$ & 9.033 & 10.338 & 4.040 & 4.701 & 19.220 & 2.650 \\
\hline $2^{21}$ & 15.794 & 12.300 & 5.194 & 7.839 & 36.470 & 4.310 \\
\hline $2^{22}$ & 15.661 & 14.138 & 7.456 & 14.254 & 71.342 & 7.662 \\
\hline $2^{23}$ & 15.053 & 16.320 & 11.999 & 27.1798 & 139.110 & 14.505 \\
\hline $2^{24}$ & 16.036 & 17.480 & 20.913 & 53.415 & 304.978 & 28.348 \\
\hline $2^{25}$ & 23.632 & 31.100 & 39.653 & - & 573.287 & 56.360 \\
\hline $2^{26}$ & 30.764 & 56.451 & 76.051 & - & - & - \\
\hline $2^{27}$ & 55.126 & 92.363 & 149.896 & - & - & - \\
\hline $2^{28}$ & 103.174 & 178.439 & - & - & - & - \\
\hline $2^{29}$ & 204.072 & - & - & - & - & - \\
\hline
\end{tabular}

borders too. In the last step, each GPU sends its three values (quantity of maximum subsequence sum, longest subsequence, and shortest subsequence) to the host that will compute the final solution.

Now we describe some strategies that we used in the implementation. To make a better use of the GPU, two techniques were utilized: persistent threads and efficient communication among SPs. A kernel was launched with the number of blocks of threads as a multiple of the amount of blocks executed concurrently in each SM. Since a SM can process one or more blocks of threads simultaneously, we avoid blocks of threads staying in the scheduling queue. As a consequence, each block of threads stay persistent and can operate on more than one part of the input, when this input is larger than the grid of blocks of the kernel. The multiplicity of the amount of blocks of threads selected was two times the number of SMs. This choice was based on the experiments using the profile nvprof, that obtained the best result, achieving $95 \%$ of occupancy.

The use of efficient communication among the SPs is related to the SIMD functionality offered by the latest versions of GPUs. This functionality allows that SPs within an SM communicate through the shared memory and

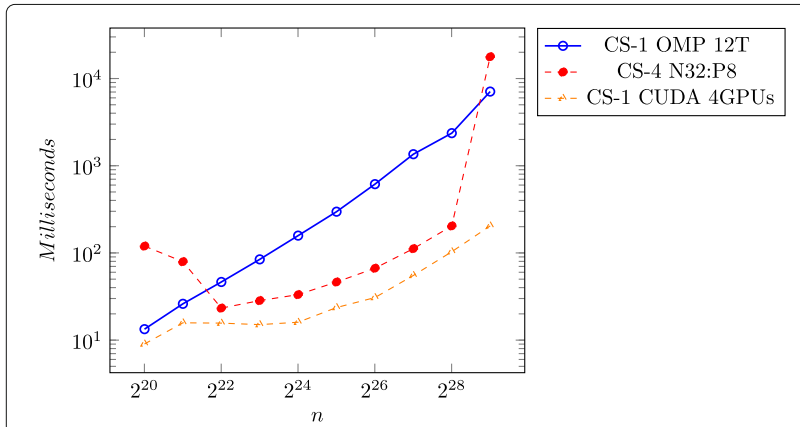

Fig. 11 Best running time (milliseconds) of each implementation without synchronization, simply using registers. A second implementation was created for older versions of GPUs (capability <3.0) by using communication through the shared memory and synchronizations.

We also exploited the memory hierarchy of the GPU by moving data from global memory to shared memory (and registers) so as to promote coalesced access and avoid the high costs associated with global memory. The thread's resource utilization was kept low, thus permitting more threads to be used per block and a full occupation the GPU SMs.

Finally, the optimized thrust::reduce was used to perform the reductions between blocks, finding the results for a single GPU. Each GPU sent its results to host for final reductions. It was possible to take advantage of memory coalescing and thereby achieve good performance results.

The running times of the CUDA GPU implementations are presented in Fig. 10 and Table 8. As in the MPI implementation, it is possible to observe some overhead for the first inputs when more than one GPU is used in the execution. However, starting from the input size $n=2^{24}$, we can note that this overhead decreases significantly. A curiosity relative to the other implementations is the fact that the

Table 9 Best running time (milliseconds) of each implementation

\begin{tabular}{llll}
\hline$n$ & CS-1 OMP 12T & CS-4 N32:P8 & CS-1 CUDA 4GPUs \\
\hline $2^{20}$ & 13.353 & 119.741 & 9.033 \\
$2^{21}$ & 26.081 & 79.335 & 15.794 \\
$2^{22}$ & 46.429 & 23.279 & 15.661 \\
$2^{23}$ & 84.330 & 28.399 & 15.053 \\
$2^{24}$ & 157.892 & 33.242 & 16.036 \\
$2^{25}$ & 297.038 & 46.379 & 23.632 \\
$2^{26}$ & 614.651 & 66.719 & 30.764 \\
$2^{27}$ & 1355.126 & 111.906 & 55.126 \\
$2^{28}$ & 2362.273 & 203.811 & 103.174 \\
$2^{29}$ & 7082.654 & $17,766.883$ & 204.072 \\
\hline
\end{tabular}




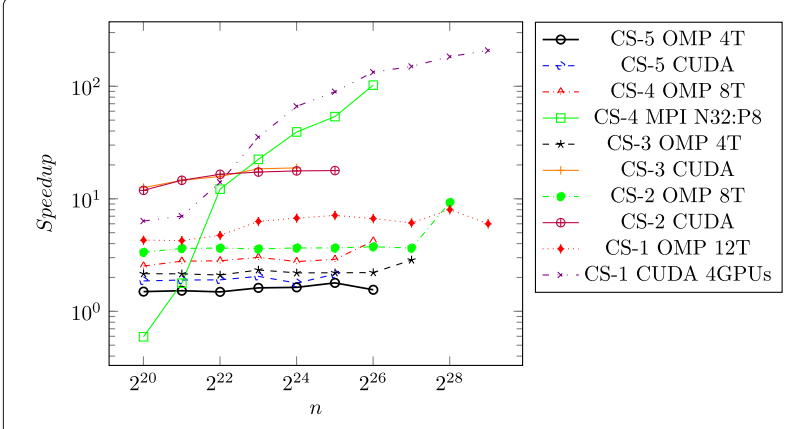

Fig. 12 Speedups - parallel/sequential in the same platform

thrashing phenomenon does not occur here. The explanation for this is that the memory of the GPUs is stressed in this situation and, on reaching the limit of its capacity, the GPU driver informs the impossibility to continue and the execution is aborted. Therefore, the main memory, in those positions used by the application, is not paged and, for this reason, the thrashing phenomenon is not verified.

\section{Best running times of each implementation and speedups}

Figure 11 and Table 9 present the best running times for each of the implementations (OpenMP, MPI, and CUDA). Since the configurations of the Platform CS-1 is a 4-GPUs system, we can already expect that the best running times of the implementations occur in this environment. The results show the scalability of the algorithm and the implementations presented: the addition of more threads/nodes/GPUs tends to present superior results.

In order to show the speedups, we computed them with respect to each platform. Figure 12 and Tables 10 and 11 present the speedups relative to the sequential and parallel implementation executed in the same platform. We can see that the OpenMP implementations, in general, tend to preserve the speedup with little variation, as well as the CUDA implementations with GPUs cards of lower performance. The exceptions are implementations CS-1 CUDA 4GPUs and CS-4 N32:P8, for which we can observe speedups of approximately 102 times for the MPI implementation and approximately 208 times for the multi-GPU CUDA version.

The platforms and equipments available were not sufficient to discover how many MPI processes (nodes and processes per node) would be sufficient to reach the speedup of the Multi-GPU implementation, due to the limitations in the submission of jobs to the cluster.

All source codes of the implementations can be found at https://github.com/rodrigogbranco/extendedmss.

\section{Conclusions}

There are good sequential and parallel solutions to the problem of maximum subsequence in the literature. However, they consider only one subsequence of maximum sum for each input sequence. Therefore, to circumvent this shortcoming, we developed BSP/CGM parallel algorithms that consider the simultaneous existence of more than one maximum subsequence sum.

Furthermore, our algorithms can find solutions for three new related problems: the maximum longest subsequence sum, the maximum shortest subsequence sum, and the number of disjoints subsequences of maximum sum. To the best of our knowledge, there are no parallel algorithms for these related problems. The algorithms work for shared and distributed memory and were implemented using a multi-GPU/CUDA, OpenMP, and MPI.

In the design of our algorithms, we use the BSP/CGM model of parallel computing. It is well known that this model is suitable for designing parallel algorithms for distributed memory platforms. In this work, we showed good results when mapping our BSP/CGM algorithms to shared memory platforms. The implementations of the proposed algorithms were shown to be efficient and have good speedup results, as confirmed by experimental results. The running times of the MPI and CUDA

Table 10 CS-4 running times (milliseconds) of MPI implementation

\begin{tabular}{|c|c|c|c|c|c|c|}
\hline$n$ & CS-5 OMP 4T & CS-5 CUDA & CS-4 OMP 4T & CS-4 N32:P8 & CS-3 OMP 4T & CS-3 CUDA \\
\hline $2^{20}$ & 1.497 & 1.863 & 2.526 & 0.594 & 2.159 & 12.570 \\
\hline $2^{21}$ & 1.525 & 1.898 & 2.792 & 1.780 & 2.153 & 14.572 \\
\hline $2^{22}$ & 1.489 & 1.904 & 2.811 & 12.188 & 2.100 & 15.803 \\
\hline $2^{23}$ & 1.615 & 2.044 & 2.811 & 22.413 & 2.330 & 18.474 \\
\hline $2^{24}$ & 1.633 & 1.78 & 3.019 & 39.274 & 2.195 & 18.824 \\
\hline $2^{25}$ & 1.789 & 2.137 & 2.770 & 53.748 & 2.201 & - \\
\hline $2^{26}$ & 1.554 & - & 2.897 & 102.308 & 2.209 & - \\
\hline $2^{27}$ & 1.651 & - & 4.212 & - & 2.858 & - \\
\hline $2^{28}$ & - & - & - & - & - & - \\
\hline $2^{29}$ & - & - & - & - & - & - \\
\hline
\end{tabular}


Table 11 Speedups - parallel/sequential in the same platform - ॥

\begin{tabular}{lllll}
\hline$n$ & CS-2 OMP 8T & CS-2 CUDA & CS-1 OMP 12T & $\begin{array}{l}\text { CS-1 CUDA } \\
\text { 4GPUs }\end{array}$ \\
\hline $2^{20}$ & 3.348 & 11.893 & 4.292 & 6.344 \\
$2^{21}$ & 3.621 & 14.610 & 4.249 & 7.017 \\
$2^{22}$ & 3.650 & 16.508 & 4.7322 & 14.030 \\
$2^{23}$ & 3.587 & 17.289 & 6.302 & 35.305 \\
$2^{24}$ & 3.657 & 17.707 & 6.738 & 66.345 \\
$2^{25}$ & 3.658 & 17.817 & 7.117 & 89.454 \\
$2^{26}$ & 3.749 & - & 6.679 & 133.460 \\
$2^{27}$ & 3.651 & - & 6.104 & 150.070 \\
$2^{28}$ & 9.288 & - & 8.014 & 183.489 \\
$2^{29}$ & - & - & 5.995 & 208.092 \\
\hline
\end{tabular}

implementations are several times better than the sequential solution in the respective platform. Furthermore, the CUDA implementation can easily be implemented with one or several GPUs.

The proposed algorithms use $p$ processors and require $\mathrm{O}(n / p)$ parallel time with a constant number of communication rounds for the algorithm of the maximum subsequence sum and $\mathrm{O}(\log p)$ communication rounds, with $\mathrm{O}(n / p)$ local computation per round, for the algorithms of the related problems.

The results obtained lead us to believe that the proposed algorithms are scalable, since the addition of processing elements in the platforms has not yet reached the saturation point. This means that the addition of threads/nodes/processors/GPUs in the respective platforms can increase the speedup significantly. Unfortunately, the available platforms do not allow us to reach the saturation point, since problems related to the limitation of the available resources have appeared, such as Thrashing and the elimination of the process by the operating system.

The results also showed the efficiency of the multiGPU version, even for large sizes of the input data. Our implementation supported up to $2^{29}$ elements with good running times, that is, supported more than 500 million elements as the input of the problem.

As future work, we intend to extend the multi-GPU implementation to solve the maximum sum subarray problem, with more than one dimension $(2 \mathrm{D}$ and $3 \mathrm{D}$ problems). We also intend to compute all the maximal subsequences in a given interval.

\section{Acknowledgements}

The authors thank FUNDECT, FAPEG, FAPESP (2013/26644-1), CNPq (482736/2012-7, 302620/2014) and Capes/PVE 002/2012 and NVIDIA. Thanks are also due to the anonymous referees for their comments and suggestions.

\section{Authors' contributions}

ACL, RAG, ENC, WSM, and SWS worked on the model proposal and the design, correctness, and complexity of the algorithms. RAG, ACL, RGB, and SF worked on the implementation details of the algorithms. All authors read and approved the final manuscript.

\section{Competing interests}

The authors declare that they have no competing interests.

\section{Author details}

${ }^{1}$ Faculdade de Computação da Universidade Federal de Mato Grosso do Sul, Cidade Universitária, C.P: 549 Campo Grande, MS, Brasil. ${ }^{2}$ Instituto de Informática da Universidade Federal de Goiás, Campus Samambaia, C.P: 131 Goiânia, GO, Brasil. ${ }^{3} I M E$, Universidade de São Paulo, Rua do Matão, São Paulo, Brasil.

Received: 8 October 2015 Accepted: 6 September 2016

Published online: 28 October 2016

\section{References}

1. Alves CER, Cáceres EN, Song SW (2004) BSP/CGM algorithms for maximum subsequence and maximum subarray. In: Recent Advances in Parallel Virtual Machine and Message Passing Interface, volume 3241 of Lecture Notes in Computer Science. Springer, Berlin Heidelberg. pp 139-146

2. Bentley J (1984) Programming pearls: algorithm design techniques. Commun ACM 27(9):865-873

3. Dehne F, Fabri A, Rau-chaplin A (1994) Scalable parallel computational geometry for coarse grained multicomputers. Int J Comput Geom 6:298-307

4. Dehne F, Ferreira A, Cáceres EN, Song SW, Roncato A (2002) A efficient parallel graph algorithms for coarse grained multicomputers and BSP. Algorithmica 33(2):183-200

5. Denning PJ (1968) Thrashing: its causes and prevention. In: Proceedings of the December 9-11, 1968, Fall Joint Computer Conference, Part I. AFIPS '68 (Fall, part I). ACM, New York. pp 915-922. doi:10.1145/1476589.1476705

6. Hwu W-MW (2011) GPU Computing Gems Jade Edition. 1. Morgan Kaufmann Publishers Inc, San Francisco

7. Ladner RE, Fischer MJ (1980) Parallel prefix computation. J ACM 27(4):831-838

8. Lima AC, Branco RG, Cáceres EN, Gaioso RA, Ferraz S, Martins WS, Song SW (2015) Efficient BSP/CGM algorithms for the maximum subsequence sum and related problems. Int Conf Comput Sci ICCS:2754-2758

9. Marcus SL, Brumell SL, Pfeifer CG, Finlay BB (2000) Salmonella pathogenicity islands: big virulence in small packages. Microbes Infect 2(2):145-156

10. Perumalla K, Deo N (1995) Parallel algorithms for maximum subsequence and maximum subarray. Parallel Process Lett 5:367-363

11. Qiu K, AkI SG (1999) Parallel maximum sum algorithms on interconnection networks. Technical report, Queens University Dept. of Com., Ontario

12. Ruzzo WL, Tompa M (1999) A linear time algorithm for finding all maximal scoring subsequences. In: Proceedings of the 7th International Conference on Intelligent Systems for Molecular Biology. AAAI Press. pp 234-241

13. Sanders J, Kandrot E (2011) CUDA by example: an introduction to general-purpose GPU programming. Ed 1. Addison-Wesley, USA

14. Shapiro SS, Wilk MB (1965) An analysis of variance test for normality (complete samples). Biometrika 3-4(52):591-611

15. Valiant LG (1990) A bridging model for parallel computation. Commun ACM 33:103-111

16. Wen Z (1995) Fast parallel algorithms for the maximum sum problem Parallel Comput 21(3):461-466 